\title{
Electronic Transport of Zigzag Graphene Nanoribbons with Edge Hydrogenation and Oxidation
}

\author{
Can $\mathrm{Cao}^{1}$, Lingna Chen ${ }^{1,2}$, Weirong Huang ${ }^{3}$ and Hui $\mathrm{Xu}^{*}{ }^{* 1}$ \\ ${ }^{I}$ School of Physics Science and Technology, Central South University, Changsha 410083, People's Republic of China \\ ${ }^{2}$ School of Computer Science and Technology, University of South China, Hengyang 421001, People's Republic of \\ China \\ ${ }^{3}$ School of Physics and Electronic Science, Changsha University of Science and Technology, Changsha 410004, \\ People's Republic of China
}

\begin{abstract}
By using non-equilibrium Green's functions in combination with the density-functional theory, we study the effect of zigzag graphene nanoribbons with edge hydrogenation and oxidation on transport properties. We find that for the ferromagnetic (FM) configuration the ZGNRs with $\mathrm{CH} 2-\mathrm{CH}$ group exhibit spin diode effect in which only one spin can occur under positive bias while the other spin occurs under negative bias. In the antiferromagnetic (AF) state the symmetric ZGNRs with CH2-CH group show the spin filter effect within some specific energy windows. However, the asymmetric ZGNRs with $\mathrm{CH} 2-\mathrm{CH}$ group do not show such a spin filter effect. We also find that the symmetric and asymmetric ZGNRs with $\mathrm{C} 2 \mathrm{O}-\mathrm{CH}$ group in AF configurations show similar transport behaviors at the Fermi level. Such ZGNRs might be exploited in spintronic nanodevices.
\end{abstract}

PACS Number(s): 71.15. Mb, 72.25.-b, 73.63.-b.

Keywords: Transport properties, spin-diode effect, first-principles, spin filter.

\section{INTRODUCTION}

Graphene Nanoribbons (GNR), quasi-one dimensional structures with a width typically smaller than $100 \mathrm{~nm}$ have attracted intensive attention in recent years due to their unique electronic properties and promising applications in nanoelectronic devices [1-5]. The electronic properties of GNRs are sensitive to many factors, such as doping [6-10], defects [11-13], edge modification [14-16], molecule adsorption [17-20], and external electrical field [21]. These provide plenty of opportunities for tuning and greatly enlarge the applications of GNRs. Among the potential uses of GNRs, recent studies have investigated the transport properties of zigzag graphene nanoribbons (ZGNRs) and the possibility of controlling their spin conductance. The edge states in ZGNRs lead to magnetic structures which are ferromagnetic along the ribbons and antiferromagnetic across the ribbons. Theory predicts edge modification to modulate the spin conductance.

The experiment and theory analyses show that the edge modification of ZGNRs is the crucial role for edge magnetism [22]. Several studies concentrate on Hydrogen $(\mathrm{H})$ termination at the edges as this seems to be one of the most stable configurations owing to its simple planar structure and it is found that a spin-polarized solution gives a lower total energy compared to a spin-degenerate calculation. But, the edge

*Address correspondence to this author at the School of Physics Science and Technology, Central South University, Changsha 410083, People's Republic of China; Tel: +86 731 88836762; Fax: +86 731 88830802; E-mail: linda_cjx@163.com passivation by $\mathrm{H}$ is not comprehensive enough to cover the realistic edge chemistry. More edge chemistry effects should be considered for more realistic modeling of GNRs. Biplab et al., [23] studied the edge passivation by $\mathrm{H} 2$, and found each edge $\mathrm{C}$ atom of ZGNR bonded with two $\mathrm{H}$ atoms open up a gap and destroy magnetism for small widths of the nanoribbon. Lee et al., [24] calculated the electronic band structures of $\mathrm{H}$ or $\mathrm{H} 2$ edge-hydrogenated zigzag graphene nanoribbons (ZGNRs) as well as $\mathrm{C} 2 \mathrm{O}$ edge-oxidized ZGNRs, and found that the eteher $(\mathrm{C} 2 \mathrm{O})$ group results in band structures similar to those of $\mathrm{sp} 3$ hybridization of $\mathrm{H} 2$ edge. However, those studies mainly focus on the electronic band structures of the edge chemistry ZGNRs, the transport property have not been investigated in details yet.

Aiming to provide a deep research, in this paper, we investigate the transport property of ZGNRs with edgechemistry modifications by $\mathrm{H}$ and $\mathrm{O}$ atoms, using the $a b$ initio DFT method within LSDA. We first study the transport property of the ZGNR with $\mathrm{H}$ and $\mathrm{H} 2$ passivation, and then consider the hydrogenation and oxidation at two edges. We mainly show that how the edge-chemistry modifications affect the transport properties of the ZGNR. Hence, the understanding of the transport properties of ZGNRs is quite important to build up electronic devices, such as spin diode [25] and spin filter [26].

\section{COMPUTATIONS}

Transport calculations are carried out within the framework of density functional theory (DFT) combined with nonequilibrium Green's function (NEGF) method as 
implemented in the Atomistix ToolKit (ATK) software package [27-28]. The two probes molecular device includes three regions: the left electrode, the right electrode, and the central scattering region. Under the applied bias $V_{b}$, the electrochemical potentials of the left and right electrode will be shifted up and down, respectively (namely, $\mu_{L}\left(V_{b}\right)=\mu_{L}(0)+e V_{b} / 2$ and $\left.\mu_{R}\left(V_{b}\right)=\mu_{R}(0)-e V_{b} / 2\right)$. Therefore, the spin-dependent current $I_{\sigma}$ through the scattering region can be calculated by Landauer-Büttiker formula [29].

$$
I_{\sigma}\left(V_{b}\right)=\frac{2 e}{\hbar} \int_{\mu_{L}\left(V_{b}\right)}^{\mu_{R}\left(V_{b}\right)} T_{\sigma}\left(E, V_{\sigma}\right) d E
$$

where the $\sigma$ represent $\operatorname{spin}-u p(\uparrow)$ and $\operatorname{spin}-\operatorname{down}(\downarrow)$. For every spin state the electron transmission coefficient $T_{\sigma}\left(E, V_{b}\right)$ is given by

$$
T_{\sigma}\left(E, V_{b}\right)=\operatorname{Tr}\left[\Gamma_{L}\left(E, V_{b}\right) G^{R}(E) \Gamma_{R}\left(E, V_{b}\right) G^{A}(E)\right]
$$

where $G^{R(A)}$ is the retarded (advanced) Green's function of the scattering region $\Gamma_{L(R)}$ is the contact broadening functions associated with the left (right) electrodes.

Our calculations have been performed by applying ATK. The local spin density approximation (LSDA) proposed by Perdew and Zunger is adopted for exchange and correlation functional, and the single- $\xi+$ polarization basis set is used for electron wave function which has been proved to well describe the $\pi$-conjugated bond system. All configurations are relaxed until their force tolerance being less than 0.05 $\mathrm{eV} / \AA$. The transport calculation includes transmission and current voltage characteristics. A cutoff energy of 150 Ry and a Monkhorst-Pack $k$ mesh of $1 \times 1 \times 100$ yield a good balance between computational time and accuracy in the results.

The model structure is constructed as follows: the scattering region is seamlessly connected to two semiinfinite ZGNR of the same width. N-ZGNRs are characterized by the number of carbon atoms along the width of the ribbon, even (symmetric) or odd (asymmetric), while Fig. (1) shows the case of eight carbon atoms abbreviated as 8- ZGNR. In order to investigate the effects of edgechemistry modifications on the transport properties, the edge $\mathrm{C}$ atoms of both lead and scattering region are terminated by additional $\mathrm{H}$ or $\mathrm{O}$. We consider three kinds of chemical edge modification: single hydrogen $(\mathrm{CH})$, double hydrogen $(\mathrm{CH} 2)$, and eteher $(\mathrm{C} 2 \mathrm{O})$, as shown in Fig. (1).

\section{RESULTS AND DISCUSSION}

We firstly show the transport properties of ZGNR with edge hydrogenation structures shown in Fig. (1a). In the Fig. (2), we show the conductance of ZGNR for two types of spin configurations which are FM (ferromagnetic spin orderings at each edge and parallel spin orientation between both the edges) and AF (ferromagnetic spin ordering at each edge and antiparallel spin orientation between both the edges). This spin-polarized ground state of ZGNRs is characterized by (a)
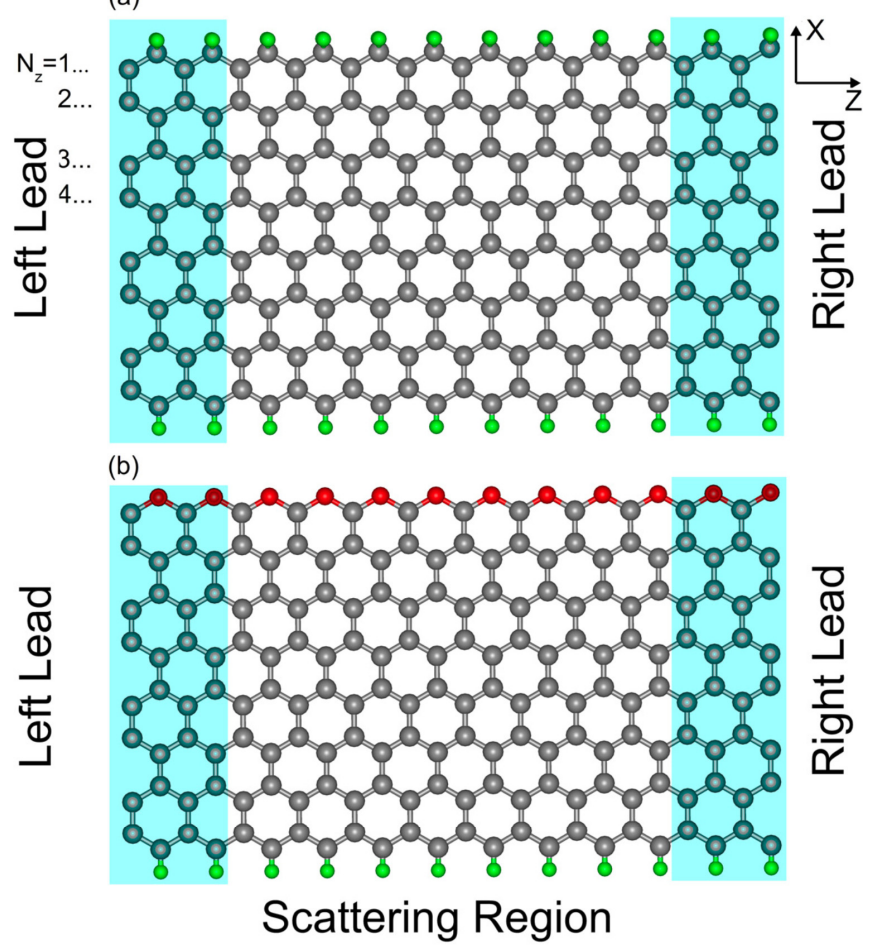

Fig. (1). (Color online) The geometry structure of the transport systems (a) with $\mathrm{CH} 2$ groups at one edge and $\mathrm{CH}$ group at the other edge, (b) with $\mathrm{C} 2 \mathrm{O}$ group at one edge and $\mathrm{CH}$ group at the other edge. The shadowed areas indicate the electrodes. The region between the shadowed areas is the scattering region.

antiparallel spin orientations of localized electronic states at the two edges of the GNR. The energy difference of ZGNR with $\mathrm{C} 2 \mathrm{O}-\mathrm{CH}$ group between the $\mathrm{AF}$ ground state and the FM state is similar to that of ZGNR with $\mathrm{CH}-\mathrm{CH}$ group, so the ZGNR with $\mathrm{C} 2 \mathrm{O}-\mathrm{CH}$ group in $\mathrm{FM}$ and $\mathrm{AF}$ configurations are stable. However, the ZGNR with $\mathrm{CH} 2-$ $\mathrm{CH}$ group is found to be less stable than ZGNR with $\mathrm{CH}-\mathrm{CH}$ group. The spin magnetization is itinerant at the edge carbon atoms of ZGNR, arising from the hybridization of $s / p$ orbital of the H/O atom with $\pi$ orbital of $\mathrm{C}$ atom. Cervantes-Sod et al., [20] found that chemical modifications of zigzag ribbons can break the spin degeneracy. Li et al., [30] found that asymmetric ZGNRs behave as conventional conductors with linear current-voltage dependence, while symmetric ZGNRs exhibit unexpected very small currents with the presence of a conductance gap around the Fermi level. Ren et al., [31] found that asymmetric ZGNR displays monotonic transport behavior, and negative differential resistance is observed in symmetric ZGNRs systems. Motivated by the above mentioned studies, we propose an alternative way to realize the spin polarization in the symmetric and asymmetric ZGNR. The transmission function for ZGNR with $\mathrm{CH}$ group at one edge and $\mathrm{CH} 2$ group at the other edge is shown in Fig. (2). It follows that the different spin of the localized state experience a different interaction with the edge state, which results in different conductance at the Fermi level in FM and AF configurations. From Fig. (2a), we can see that the transmission of 8-ZGNRs for FM shows semiconductor characteristic which is 
consistent with the band structure calculation reported by Lee et al., [24]. Interestingly, for FM we find that spin-up conductance is allowed whereas spin-down conductance is suppressed with the energy window from -0.5 to $0 \mathrm{eV}$, while for the energy window from 0 to $0.55 \mathrm{eV}$ the opposite spin species conductance is allowed or suppressed. It is a spindiode effect that only one spin channel is active and the other spin channel is closed at positive bias, and the opposite case occurs for negative bias. For AF cases, since two spin channels of 8-ZGNRs are affected differently, a high transmission peak for spin-up appears below the Fermi level, and the conductance of the spin-up near the Fermi level rises from $1 \mathrm{G}_{0}$ to $3 \mathrm{G}_{0}$, as shown in Fig. (2b). The effect of symmetric ZGNRs for AF is expect that the spin-up channel is selected while the spin-down channel is blocked due to extra $\mathrm{H}$ atom added to one edge of nanoribbons. As a result, the 8-ZGNRs with $\mathrm{CH} 2-\mathrm{CH}$ group may act as a spin filter within some specific energy windows. Fig. (2c) shows the conductance of 9-ZGNR for FM configuration. We observe that in the FM configuration the electrons with one spin
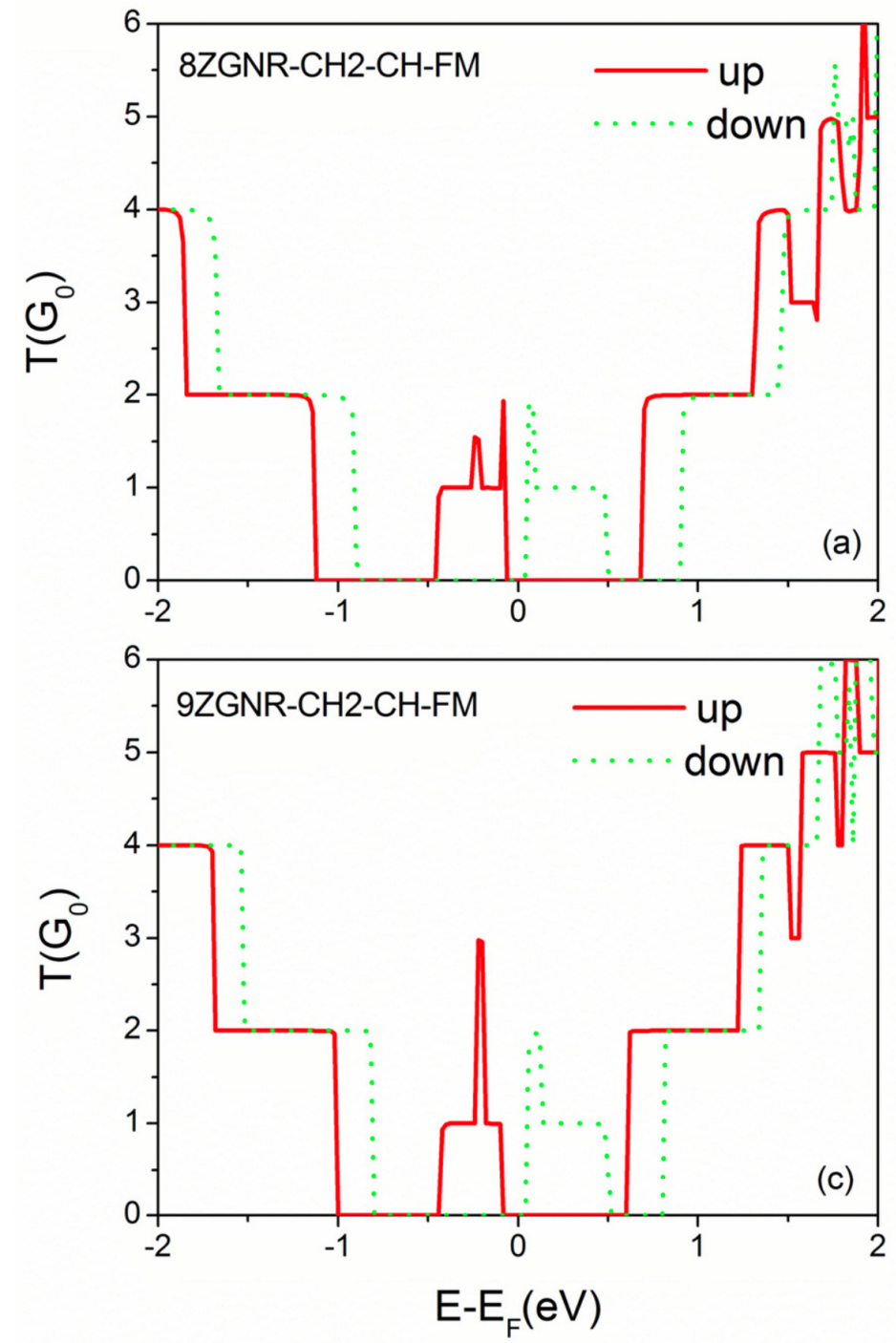

orientation will be filtered out while the other spin electrons will be almost full transmitted within the energy range from 0.5 to $0.55 \mathrm{eV}$. The effect we propose here is a spin-diode. From Fig. (2d), we find that for AF configuration of 9ZGNR the height of transmission peak of spin-up reaches $2 \mathrm{G}_{0}$ near the Fermi level. By comparing the transmissions both $9-\mathrm{ZGNR}$ and $8-\mathrm{ZGNR}$ with $\mathrm{CH} 2-\mathrm{CH}$ group, it can be seen that the symmetry of the nanoribbon width is dominant factor that affects the transmission results. Due to the sp3 bonding of the edge atom, in the FM state the symmetric and asymmetric ZGNRs with $\mathrm{CH} 2-\mathrm{CH}$ group both exhibit the spin-diode effect.

Secondly, we investigate the spin transport of ZGNR with oxidation at one edge and hydrogenation at the other edge (C2O-CH) shown in Fig. (1b). Fig. (3) shows representative conductance results for 8-ZGNRs and 9ZGNRs with two spin configuration. As can be see from the Fig. (3a), there is no transmission gap near the Fermi level. At the Fermi level the conductance of the spin-up is $1 \mathrm{G}_{0}$ while the conductance of the spin-down is $2 \mathrm{G}_{0}$. In this
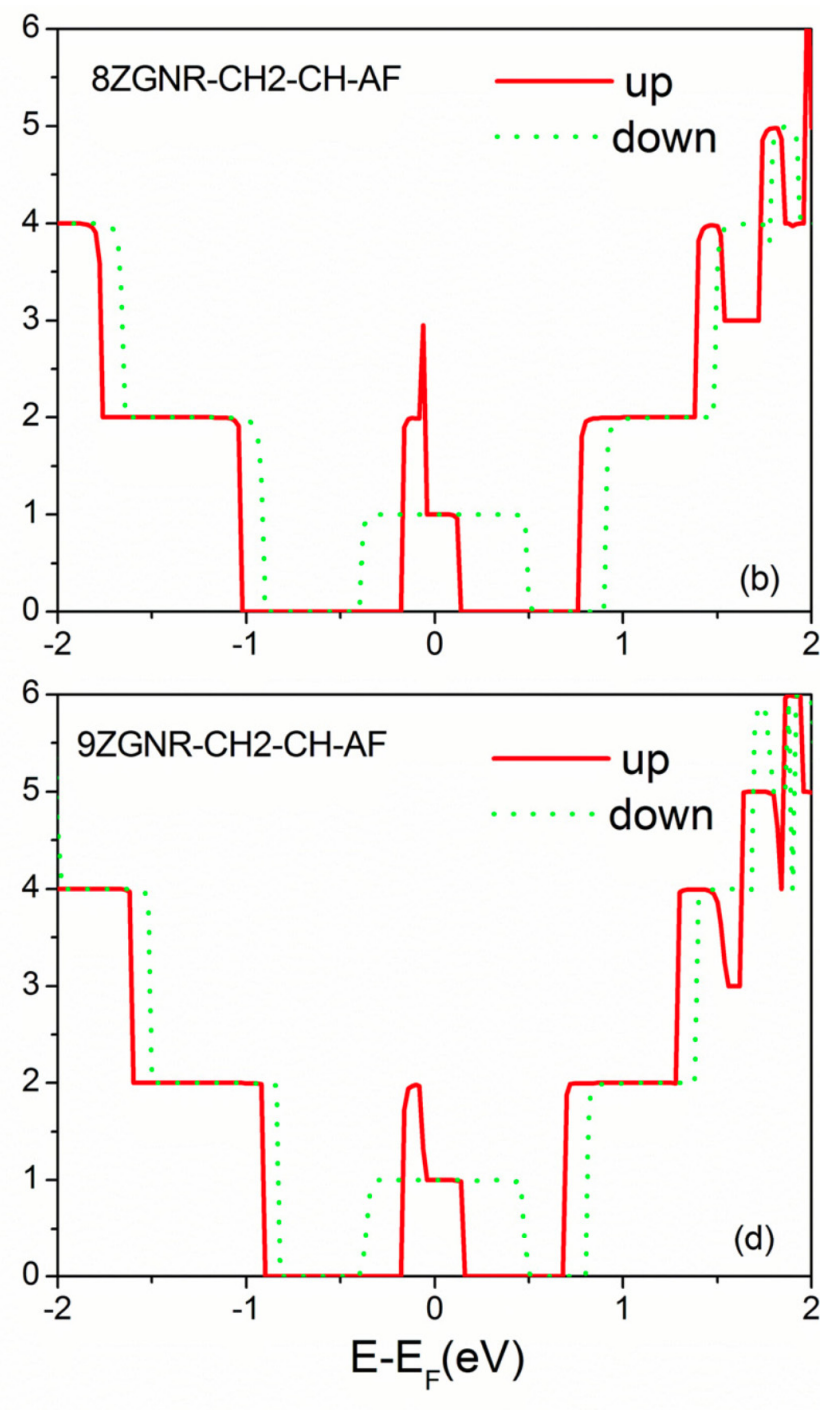

Fig. (2). (Color online) Transmission functions for (a) 8-ZGNR with $\mathrm{CH} 2-\mathrm{CH}$ group for FM configuration, (b) 8-ZGNR with $\mathrm{CH} 2-\mathrm{CH}$ group for AF configuration, (c) 9-ZGNR with $\mathrm{CH} 2-\mathrm{CH}$ group for FM configuration, (d) 9-ZGNR with $\mathrm{CH} 2-\mathrm{CH}$ group for AF configuration. Spin-up and down states are represented by the red solid and green dotted lines in the transmission. 

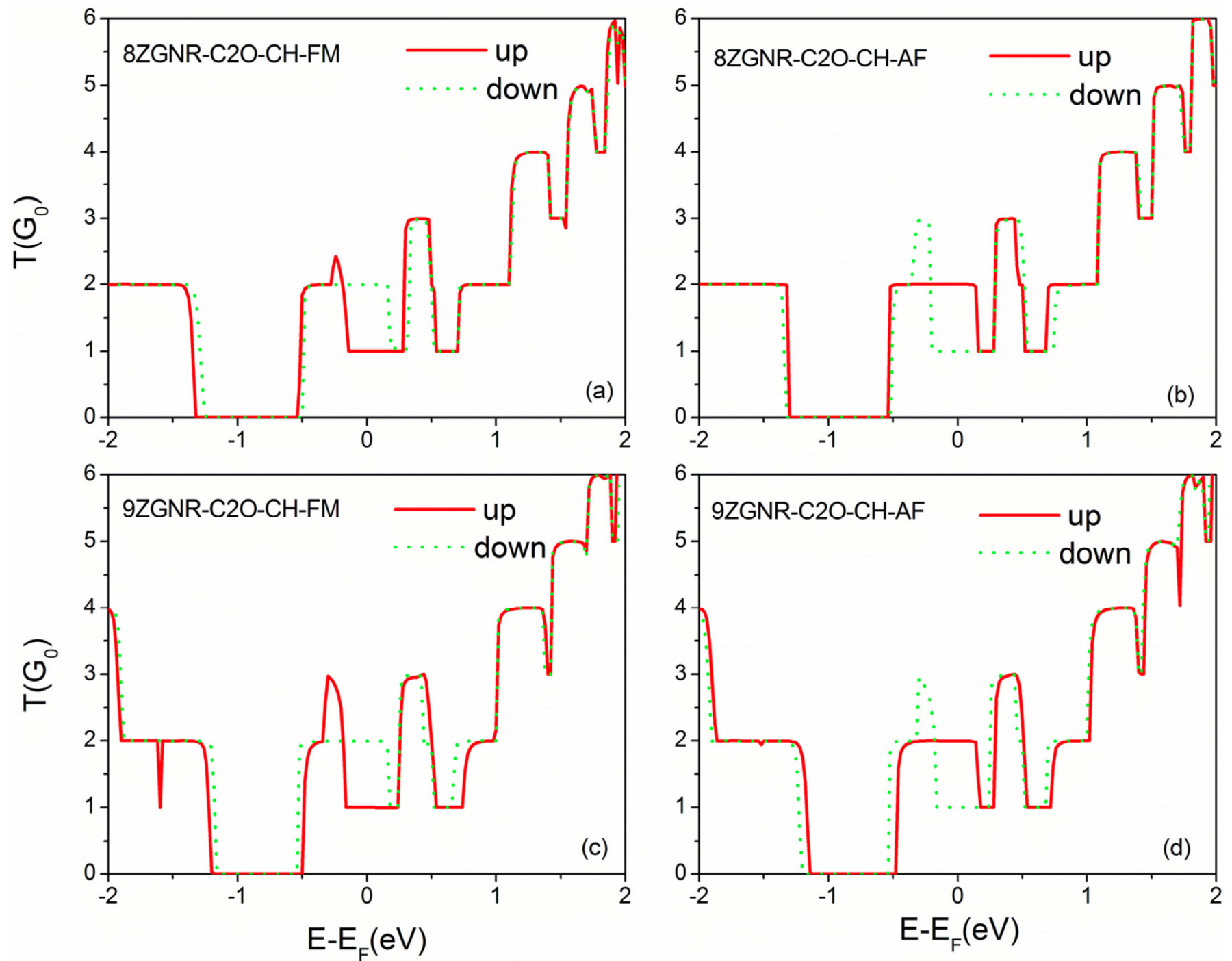

Fig. (3). (Color online) Transmission functions for (a) 8-ZGNR with C2O-CH group for FM configuration, (b) 8-ZGNR with C2O-CH group for AF configuration, (c) 9-ZGNR with $\mathrm{C} 2 \mathrm{O}-\mathrm{CH}$ group for FM configuration, (d) 9-ZGNR with $\mathrm{C} 2 \mathrm{O}-\mathrm{CH}$ group for AF configuration. Spin-up and down states are represented by the red solid and green dotted lines in the transmission.

situation the energy of edge state is shifted towards the valence bands due to the oxidation at one edge. From Fig. (3b), we find that for AF configuration the height of the transmission peak of 8-ZGNRs with $\mathrm{C} 2 \mathrm{O}-\mathrm{CH}$ group rises below the Fermi level. The rise can be correlated with the oxygen atom induced states and the peak to edge-related states. As the carbon atom at the edge is substituted by the oxygen atom, two spins of 9-ZGNRs with $\mathrm{C} 2 \mathrm{O}-\mathrm{CH}$ group split at the Fermi level, leading zero transmissions gap, as shown in Fig. (3c). A similar behavior is predicted for the AF configuration of 9-ZGNRs with $\mathrm{C} 2 \mathrm{O}-\mathrm{CH}$ group (Fig. 3d), and the transmission peak correspond the strongly localized on the edge of ZGNRs. This means that the proposed use of ZGNR with $\mathrm{C} 2 \mathrm{O}-\mathrm{CH}$ group as chemical sensors may be possible due to edge oxidation.

Thirdly, we consider the spin-polarized current in the symmetric and asymmetric ZGNRs junction as a function of the applied bias. For 8-ZGNRs and 9-ZGNRs we focus spin configuration with an FM and AF junction. The spin configuration is crucial for spin filter effect we discuss, and might be realized by chemical modification for ZGNRs. Fig. (4) shows the $I-V$ curves of ZGNRs with $\mathrm{CH} 2-\mathrm{CH}$ and $\mathrm{C} 2 \mathrm{O}$ $\mathrm{CH}$ group under the $\mathrm{FM}$ and $\mathrm{AF}$ configuration. As shown in Fig. (4a), the current of spin-up and spin-down is degenerate in the symmetric and asymmetric ZGNRs for $\mathrm{CH} 2-\mathrm{CH}$ group under FM configuration. For AF configuration, the current of spin-up decrease obviously with the increase of the bias while the current of spin-down is always zero when the bias takes a value between $|V|=0.3 \mathrm{~V}$ and $|V|=0.8 \mathrm{~V}$. So the spinup electrons can flow while the spin-down electrons are fully reflected in this bias range. The effect can be regard as a spin filter under certain bias range. But for 9-ZGNRs with $\mathrm{CH} 2-$ $\mathrm{CH}$ group under AF configuration as described in Fig. (4b), the current of spin-up and spin-down is the slightly different when bias is larger than 0.4 V. From Fig. (4), it can be found that the obvious negative differential resistance (NDR) behavior appears in the symmetric and asymmetric ZGNRs with $\mathrm{C} 2 \mathrm{O}-\mathrm{CH}$ group. From Fig. (4c), we can see clearly that in the bias range from $|V|=0.5$ to $|V|=0.9 \mathrm{~V}$ the spin current 

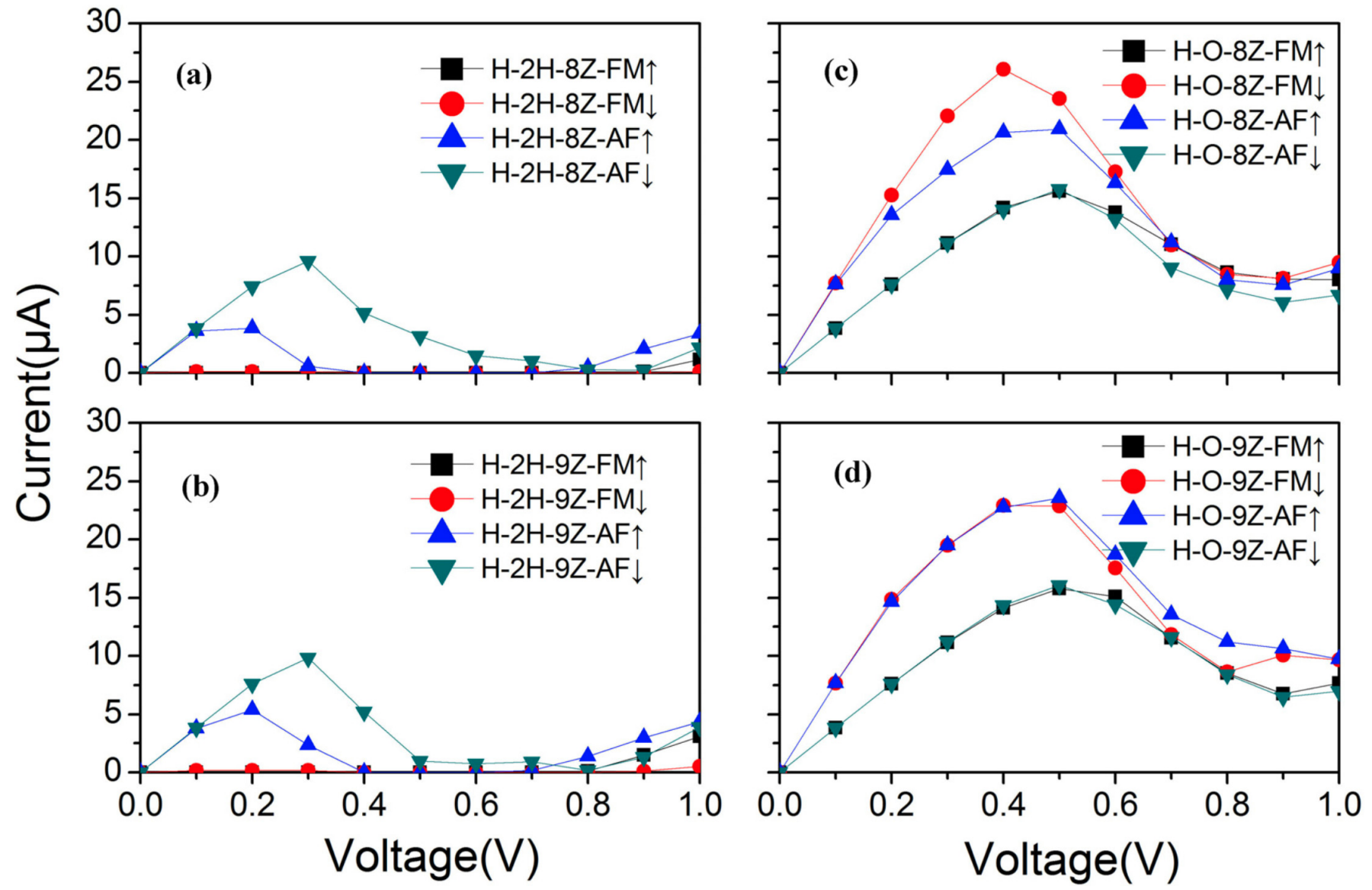

Fig. (4). (Color online) The spin currents as functions of the applied bias under FM and AF configurations for: (a) 8-ZGNR with CH2-CH group, (b) 9-ZGNR with CH2-CH group, (c) 8-ZGNR with C2O-CH group, (d) 9-ZGNR with C2O-CH group.

decreases with the increase of the bias under $\mathrm{AF}$ configuration, which shows NDR behavior in the bias range. Comparing Fig. (4d) with Fig. (4c), it can be found that under $\mathrm{AF}$ configuration the $I-V$ curves exhibit a similar characteristic in the symmetric and asymmetric ZGNRs with $\mathrm{C} 2 \mathrm{O}-\mathrm{CH}$ group. However, for FM configuration, we can find that the spin current with different width is different when bias is less than $0.7 \mathrm{~V}$. From these results, it is known that the edge-chemistry modifications can adjust the spin current of ZGNRs, and the symmetry plays an important role in transport properties. As we know, the magnetic symmetry of the ZGNR can be broken due to the the edge defect. The symmetric ZGNRs with edge-chemistry modifications are more favorable for spin transport in contrast to spin-split gap in the asymmetric ZGNRs.

At last, in order to explain the origin of the spin transport properties, we present molecularly projected self-consistent Hamiltonian (MPSH) of two channels within bias window at 0.3 and $0.8 \mathrm{~V}$ as shown in Fig. (5a). Note that the MPSH can help us to understand the origin of the spin transport characteristics. It is known that the main contribution to the transport comes from the frontier molecular orbital's HOMO and LUMO. From Fig. (5a), we find that HOMO and LUMO are delocalized across the whole scattering region under the $\mathrm{V}_{\mathrm{b}}=0.3 \mathrm{~V}$, which results from the strong coupling between electrodes and the scattering region. When the bias is increased up to $0.8 \mathrm{~V}$, HOMO and LUMO are almost fully localized, which leads to the decrease of current due to the strong electron backscattering. For the 8-ZGNRs with C2O$\mathrm{CH}$ group under the AF configuration as shown in Fig. (5b), in the bias range from the $V=0.3$ to $V=0.5 \mathrm{~V}$, we can see that there are more transmission coefficients entering into the bias window with the increase of bias, resulting in the increase of the current. However, in the bias range from the $V=0.5$ to $V=0.9 \mathrm{~V}$, the total integral area of transmission coefficients in the bias window becomes smaller with the increase of the bias. As a result, the current is decreased and NDR appears. We also find that when the bias is larger than $0.9 \mathrm{~V}$, other integral area enters the bias window and the current increase again and NDR behavior disappears. From the above results, we can infer that the peculiar $I-V$ curves can be determined by the spin band structure of ZGNRs and the symmetry of wave functions ${ }^{24}$. This phenomenon depict that the ZGNRs with $\mathrm{C} 2 \mathrm{O}-\mathrm{CH}$ group can be use as spintronic devices. The above results show that when the spin polarization is considered, symmetric ZGNRs with different edge-chemistry modifications present different transport behavior. Such different transport behaviors result from different coupled electronic states supported by the wave functions.

\section{CONCLUSIONS}

In conclusion, we calculate the transport properties of the symmetric and asymmetric ZGNRs with edge hydrogenation 
(a)

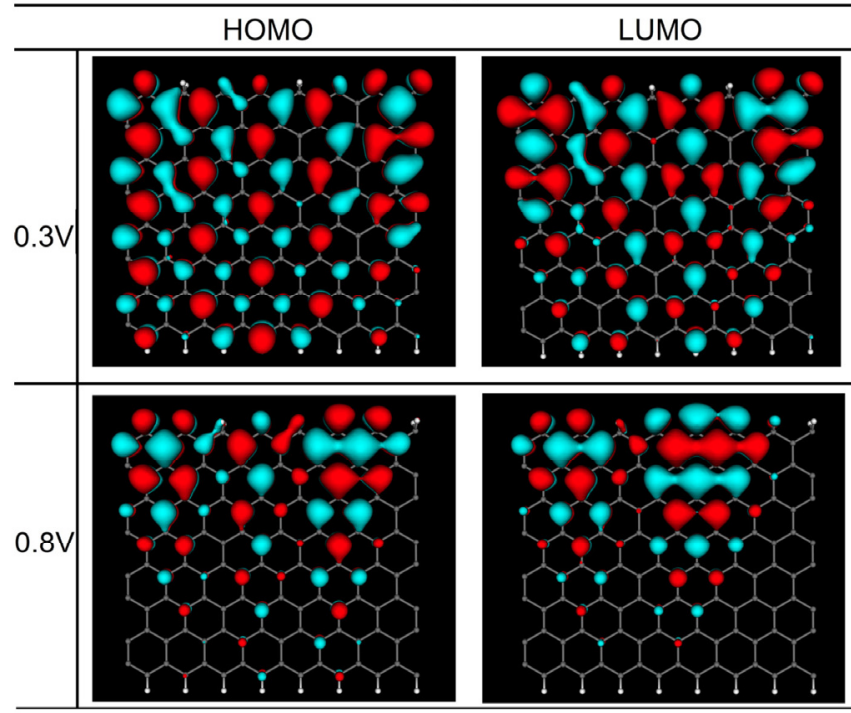

(b)

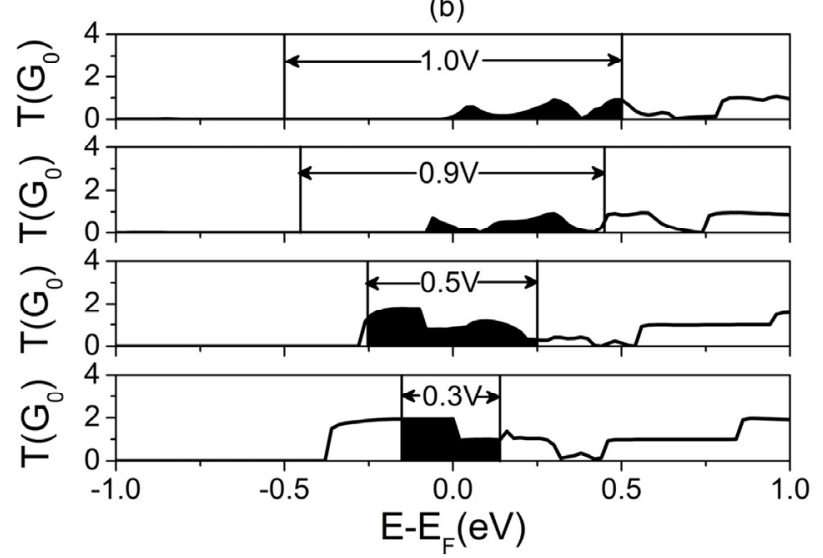

Fig. (5). (Color online) (a) The MPSH of spin down for HOMO and LUMO under bias $0.3 \mathrm{~V}$ and $0.8 \mathrm{~V}$ in the 8-ZGNR with $\mathrm{CH} 2-$ $\mathrm{CH}$ group under AF configuration, respectively. (b) The transmission functions correspond to the spin down bias of being $0.3,0.5,0.9 \mathrm{~V}, 1.0 \mathrm{~V}$ in the 8-ZGNR with $\mathrm{C} 2 \mathrm{O}-\mathrm{CH}$ group under $\mathrm{AF}$ configuration, respectively.

and oxidation using the ab initio density-functional method. The results show that for FM configuration the symmetric and asymmetric ZGNRs with $\mathrm{CH} 2-\mathrm{CH}$ group can be used as a spin-diode device due to the sp3 bonding of $\mathrm{CH} 2$ edge. For the AF magnetic structure of $\mathrm{CH} 2-\mathrm{CH}$, a spin filter within some specific energy windows can be act in the symmetric ZGNRs. The physical origin of the spin filter effect can be attributed to the spin polarized band structure of the symmetric ZGNRs near the Fermi level [24]. However, the asymmetric ZGNRs with $\mathrm{CH} 2-\mathrm{CH}$ group do not show such a spin filter effect. The symmetric and asymmetric ZGNRs with $\mathrm{C} 2 \mathrm{O}-\mathrm{CH}$ group in AF configurations show similar transport behaviors due to the metallic band caused by the $\mathrm{C}$ $\mathrm{O}$ chemical interaction. Our findings may shed light on the effect of edge-chemistry modified in the ZGNR and are helpful for devising spin devices based on magnetized graphene nanoribbon.

\section{ACKNOWLEDGEMENTS}

This work was supported by National Natural Science Foundation of China (Grant No. 50721003), the Natural Science Foundation of Hunan Province (Grant No 11JJ3073), the Scientific Research Fund of Hunan Provincial Education Department (Grant No 10C1171), and High Performance Computing Center of CSU.

\section{CONFLICT OF INTEREST}

\section{Declared none.}

\section{REFERENCES}

[1] Wang B, Wang J, Guo H. Ab initio calculation of transverse spin current in graphene nanostructures. Phys Rev B 2009; 79: $165417-$ 5 .

[2] Brito WH, Miwa RH. Adsorption and diffusion of gold adatoms on graphene nanoribbons: An ab initio study. Phys Rev B 2010; 82: 045417-7.

[3] Bhowmick S. Anisotropy of the Stone-Wales defect and warping of graphene nanoribbons: A first-principles analysis. Phys Rev B 2010; 81: 155416-7.

[4] Topsakal M, Aktürk E, Sevinçli H, Ciraci1S. First-principles approach to monitoring the band gap and magnetic state of a graphene nanoribbon via its vacancies. Phys Rev B 2008; 78: 235435-6.

[5] Chaves AJ, Lima GD, de Paula W, et al. Dynamical gap generation in graphene nanoribbons: an effective relativistic field theoretical model. Phys Rev B 2011; 83: 153405-4.

[6] Kim G, Jhi SH. Optimization of metal dispersion in doped graphitic materials for hydrogen storage. Phys Rev B 2008; 78: 085408-5.

[7] Biel B, Blase X, Triozon F, et al. Anomalous Doping Effects on Charge Transport in Graphene Nanoribbons. Phys Rev Lett 2009; 102: 096803-4

[8] Martins TB, Miwa RH, da Silva AJR, et al. Electronic and transport properties of boron-doped graphene nanoribbons. Phys Rev Lett 2007; 98: 196803-4.

[9] Bushmaker AW, Vikram VD, Hsieh S, et al. Direct observation of born-oppenheimer approximation breakdown in carbon nanotubes. Nano Lett 2009; 9: 607-11.

[10] Cervantes-Sodi F, Csányi G, Piscanec S, Ferrari AC. Edgefunctionalized and substitutionally doped graphene nanoribbons: electronic and spin properties. Phys Rev B 2008; 77: 165427-13.

[11] Savin AV, Kivshar YS. Vibrational Tamm states at the edges of graphene nanoribbons. Phys Rev B 2010; 81: 165418-9.

[12] Bradley FH, Oleg NK, Oleg VP. Ab initio study of phonon-induced dephasing of electronic excitations in narrow graphene nanoribbons. Nano Lett 2008; 8: 2510-6.

[13] Cresti1 A, Roche S. Edge-disorder-dependent transport length scales in graphene nanoribbons: from Klein defects to the superlattice limit. Phys Rev B 2009; 79: 233404-4.

[14] Cervantes-Sodi F, Csányi G, Piscanec S, Ferrari AC. Edgefunctionalized and substitutionally doped graphene nanoribbons electronic and spin properties. Phys Rev B 2008; 77: 165427-13.

[15] Wang Y, Cao C, Cheng HP. Metal-terminated graphene nanoribbons. Phys Rev B 2010; 82: 205429-5.

[16] Saloriutta K, Hancock Y, Karkkainen A, et al. Electron transport in edge-disordered graphene nanoribbons. Phys Rev B 2011; 83: 205125-6.

[17] Chowdhury R, Adhikari S, Rees P, et al. Graphene-based biosensor using transport properties. Phys Rev B 2008; 83: 045401-8.

[18] Ma J, Michaelides A, Alfe D, et al. Adsorption and diffusion of water on graphene from first principles Phys Rev B 2011; 84: 033402-4.

[19] Ihnatsenka S, Kirczenow G. Dirac point resonances due to atoms and molecules adsorbed on graphene and transport gaps and conductance quantization in graphene nanoribbons with covalently bonded adsorbates. Phys Rev B 2011; 83: 245442-19.

[20] Cervantes-Sodi F, Csányi G, Piscanec S, et al. Edge-functionalized and substitutionally doped graphene nanoribbons: electronic and spin properties. Phys Rev B 2008; 77: 165427-13. 
[21] Dutta S, Manna AK, Pati SK. Intrinsic Half-Metallicity in Modified Graphene Nanoribbons. Phys Rev Lett 2009; 102: 096601-4.

[22] Kunstmann J, Özdogan C, Quandt A, et al. Stability of edge states and edge magnetism in graphene nanoribbons. Phys Rev B 2011; 83: 045414-8.

[23] Bhandary S, Eriksson O, Sanyal B. Complex edge effects in zigzag graphene nanoribbons due to hydrogen loading. Phys Rev B 2010; 82: $165405-7$.

[24] Lee G, Cho K. Electronic structures of zigzag graphene nanoribbons with edge hydrogenation and oxidation. Phys Rev B 2009; 79: 165440-12.

[25] Martins T B, da Silva A J R, Miwa R H, et al. $\sigma$ - and $\pi$-Defects at graphene nanoribbon edges: building spin filters. Nano Lett 2008; 8: 2293-8.

[26] Ozaki T, Nishio K, Wang H, et al. Dual spin filter effect in a zigzag graphene nanoribbon. Phys Rev B 2010; 81: 074522-5.
Brandbyge $\mathrm{M}$, Mozos J, Ordejon $\mathrm{P}$, et al. Density-functional method for nonequilibrium electron transport. Phys Rev B 2002; 65: 165401-17.

[28] Qin R, Lu J, Lai L, et al. Room-temperature giant magnetoresistance over one billion percent in a bare graphene nanoribbon device. Phys Rev B 2010; 81: 233403-4.

[29] Buttiker M, Imry Y, Landauer R, et al. Generalized many-channel conductance formula with application to small rings. Phys Rev B 1985; 31: 6207-15.

[30] Li Z Y, Qian H Y, Wu J, et al. Role of symmetry in the transport properties of graphene nanoribbons under bias. Phys Rev Lett 2008; 100: 206802.

[31] Ren Y, Chen K Q. Effects of symmetry and stone-Wales defect on spin-dependent electronic transport in zigzag graphene nanoribbons. J Appl Phys 2010; 107: 044514.

(C) Cao et al.; Licensee Bentham Open.

This is an open access article licensed under the terms of the Creative Commons Attribution Non-Commercial License (http://creativecommons.org/licenses/by-nc/3.0/) which permits unrestricted, non-commercial use, distribution and reproduction in any medium, provided the work is properly cited. 